\title{
Quantum black holes from null expansion operators
}

\author{
Viqar Husain and Oliver Winkler* \\ Department of Mathematics and Statistics, University of New Brunswick, Fredericton, NB, Canada E3B 5A3
}

(Dated: October 15, 2018)

\begin{abstract}
Using a recently developed quantization of spherically symmetric gravity coupled to a scalar field, we give a construction of null expansion operators that allow a definition of general, fully dynamical quantum black holes. These operators capture the intuitive idea that classical black holes are defined by the presence of trapped surfaces, that is surfaces from which light cannot escape outward. They thus provide a mechanism for classifying quantum states of the system into those that describe quantum black holes and those that do not. We find that quantum horizons fluctuate, confirming long-held heuristic expectations. We also give explicit examples of quantum black hole states. The work sets a framework for addressing the puzzles of black hole physics in a fully quantized dynamical setting.

PACS numbers: 04.60.Ds
\end{abstract}

Black holes are probably the most fascinating prediction of general relativity. A host of observational data over the last decade has firmly established them as objects within experimental reach 1, 2]. From a theoretical point of view, they are particularly attractive in that they offer a glimpse into the as yet unknown realm of quantum gravity. Examples are the analogy of black hole mechanics with the laws of thermodynamics, Hawking radiation, and the question of the final fate of the matter whose collapse forms the black hole. The latter is closely connected with the question of what happens to the classical singularity.

While classical and semiclassical methods have yielded important insights [3], there is no doubt that investigations in a fully quantized setting are required to obtain a deeper understanding of black hole physics. As a first step in this direction, we developed a quantum theory of spherically symmetric gravity systems [4]. This has the attractive feature that the classical singularity is resolved at the quantum level. In order to use this framework for studying dynamical black holes, a practically useful definition of quantum black holes is required, as well as a criterion for selecting the associated quantum states. This is the topic we address in this Letter.

Traditionally, the notion of a black hole has been intimately connected with the idea of an event horizon. The reason is that this is a very useful concept to study static aspects of black holes, and has indeed been used to derive important results, some of which were mentioned above. Its drawback, however, is its global nature, which makes it unsuitable for investigating dynamical processes, such as gravitational collapse, black hole mergers, and black hole evaporation. To remedy this situation, more local definitions of a classical black hole have been developed. They are based on the concept of a trapped spatial 2surface.

The intuitive idea is to look at the light emanating from a surface. If no light propagates outward (defined in a suitable sense), then the surface is considered to be trapped. This criterion may be used to divide regions of space into those containing trapped surfaces, and those that do not. The outermost boundary of a trapped region may be used as a definition of the (dynamical) black hole horizon.

To make this idea more precise consider a closed embedded 2-surface $S$ with normal $s^{a}\left(s_{a} s^{a}=1\right)$ in a spatial slice $\Sigma$ of a spacetime $\left(M, g_{a b}\right)$. Let $n^{a}\left(n^{a} n_{a}=-1\right)$ denote the timelike normal to $\Sigma$ as embedded in $M$. Then we have the decompositions

$$
q_{a b}=g_{a b}+n_{a} n_{b}, \quad \sigma_{a b}=q_{a b}-s_{a} s_{b},
$$

for the induced metrics $q_{a b}$ on $\Sigma$ and $\sigma_{a b}$ on $S$. The expansions of the future pointing "outgoing" $(+)$ and "ingoing" (-) null vectors $l_{ \pm}^{a}=n^{a} \pm s^{a}$ on $S$ are defined by

$$
\theta_{ \pm}=\sigma^{a b} D_{a} l_{ \pm b}=s_{a} s_{b} \pi^{a b} \pm D_{a} s^{a}
$$

where $D_{a}$ is the $q_{a b}$ covariant derivative, and the last equality uses the Arnowitt-Deser-Misner ADM momentum variable $\pi^{a b}=K^{a b}-K q^{a b}$, where $K^{a b}$ is the extrinsic curvature of $\Sigma$ and $K$ is its trace. The surface $S$ is called outer trapped if

$$
\theta_{+} \leq 0, \quad \theta_{-}<0
$$

and marginally outer trapped if

$$
\theta_{+}=0, \quad \theta_{-}<0 .
$$

These conditions hold for generic slices of all known black hole spacetimes, and therefore provide a natural test to see if $\left(\Sigma, q_{a b}, \tilde{\pi}^{a b}\right)$ is a slice of a black hole spacetime. The time evolution of the marginal outer trapping boundary forms a 3 -surface in $M$ called the trapping horizon [5]. Unlike the event horizon, it is not necessarily a null surface. This reflects its usefulness for capturing dynamical processes which change the size and location of a horizon. The intersections of a trapping horizon with the 
spatial hypersurfaces of a canonical decomposition provide a very useful tracking mechanism for such processes in time sequence. This is routinely used in numerical evolution for example. It is also the appropriate framework for our canonical quantization program.

The system we consider is a canonical formulation of the asymptotically flat gravity in spherical symmetry. Although this system has been studied both with and without matter classically and semi-classically 6, 7, 8, 9, 10, 11, 12, 13], a complete quantization with scalar field coupling has to our knowledge never been achieved. Recently we have given a new quantization of this model in Painleve-Gullstrand coordinates [4]. Our main goal in the present work is to provide in that framework a construction of operators $\hat{\theta}_{ \pm}$corresponding to the classical expansions $\theta_{ \pm}$, and use them to identify black hole quantum states.

We first review briefly the canonical setting and its quantization, and then proceed to describe in detail a construction of expansion operators. Using these operators, we give a criterion for defining the quantum states that correspond to classically trapped configurations, and construct some explicit examples of such states.

The classical Hamiltonian theory of the spherically symmetric gravity-scalar field model used is developed in [4]. This system is adapted to the Painleve-Gullstrand (PG) coordinates, in which the black hole metric is

$$
d s^{2}=-d t^{2}+\left(d r+\sqrt{\frac{2 M}{r}} d t\right)^{2}+r^{2} d \Omega^{2} .
$$

The spatial metric $e_{a b}$ given by the constant $t$ slices is flat and the extrinsic curvature of the slices is

$$
K_{a b}=-\sqrt{\frac{2 M}{r^{3}}}\left(e_{a b}-\frac{3}{2} s_{a} s_{b}\right),
$$

where $s^{a}=x^{a} / r$ for Cartesian coordinates $x^{a}$. It is evident from this that the black hole mass information is contained only in the extrinsic curvature, which in the ADM phase space variables $\left(q_{a b}, \tilde{\pi}^{a b}\right)$ determines the momenta $\tilde{\pi}^{a b}$ conjugate to the spatial metric $q_{a b}$.

The phase space of the model is defined by prescribing a form of the gravitational phase space variables $q_{a b}$ and $\tilde{\pi}^{a b}$. Asymptotic flatness falloff conditions are then imposed on these variables, and on the lapse and shift functions $N$ and $N^{a}$, such that the canonical ADM action is well defined.

In this setting, the following parametrization for the 3 -metric and conjugate momentum gives a reduction to spherical symmetry [4]

$$
\begin{aligned}
q_{a b} & =\Lambda(r, t)^{2} s_{a} s_{b}+\frac{R(r, t)^{2}}{r^{2}}\left(e_{a b}-s_{a} s_{b}\right) \\
\tilde{\pi}^{a b} & =\frac{P_{\Lambda}(r, t)}{2 \Lambda(r, t)} s^{a} s^{b}+\frac{r^{2} P_{R}(r, t)}{4 R(r, t)}\left(e^{a b}-s^{a} s^{b}\right) .
\end{aligned}
$$

Substituting these into the $3+1 \mathrm{ADM}$ action shows that the pairs $\left(R, P_{R}\right)$ and $\left(\Lambda, P_{\Lambda}\right)$ are canonically conjugate variables. The reduced ADM $1+1$ field theory action takes the form

$$
\begin{aligned}
S_{R}= & \frac{1}{2 G} \int d t d r\left(P_{R} \dot{R}+P_{\Lambda} \dot{\Lambda}+P_{\phi} \dot{\phi}\right) \\
& + \text { constraint and surface terms. }
\end{aligned}
$$

This completes the definition of the classical theory. The two reduced constraints, the Hamiltonian and radial diffeomorphism generators are first class. A surface term is needed to ensure functional differentiability of $S_{R}$.

The classical expansions (2) in spherical symmetry are functions of the phase space variables given by

$$
\theta_{ \pm}=-\frac{1}{2 \Lambda}\left(2 R^{2} \Lambda \Lambda^{\prime} \pm P_{\Lambda}+4 \Lambda^{2} R R^{\prime}\right) .
$$

Given data $\left(R(r), P_{R}(r) ; \Lambda(r), P_{\Lambda}(r)\right)$ on a spatial hypersurface $\Sigma$, the marginal trapping horizon(s) are located by finding the solution coordinates $r=r_{i}(i=1 \cdots n)$ of Eqns. (44) (since in general there may be more than one solution). The corresponding radii $R_{i}=R\left(r_{i}\right)$ are then computed. The size of the horizon on the slice $\Sigma$ is the largest value in the set $\left\{R_{i}\right\}$.

Turning now to the quantum theory where the horizon operators corresponding to these classical conditions will be defined, let us recall the quantization used in [4]. A typical basis state is given by

$$
\begin{aligned}
& \left|e^{i \sum_{k} a_{k} P_{R}\left(x_{k}\right)}, e^{i \sum_{l} b_{l} P_{\Lambda}\left(y_{l}\right) / L}, e^{i L^{2} \sum_{m} c_{m} P_{\phi}\left(z_{m}\right)}\right\rangle \\
& \equiv\left|a_{1} \ldots a_{N_{1}} ; b_{1} \ldots b_{N_{2}}, c_{1} \ldots c_{N_{3}}\right\rangle
\end{aligned}
$$

where $a_{k} \cdots c_{m}$ are real numbers which are the analogs of the occupation numbers in a Fock space representation, and the factors of $L$ in the exponents reflect the length dimensions of the respective field variables. The inner product on this basis is given by

$$
\left\langle a_{1} \ldots c_{N_{3}} \mid a_{1}^{\prime} \ldots c_{N_{3}}^{\prime}\right\rangle=\delta_{a_{1}, a_{1}^{\prime}} \cdots \delta_{c_{N_{3}}, c_{N_{3}}^{\prime}},
$$

if the states contain the same sampled points, and is zero otherwise.

The configuration operators are defined by

$$
\hat{R}_{f}\left|a_{1} \ldots c_{N_{3}}\right\rangle=L \sum_{k} a_{k} f\left(x_{k}\right)\left|a_{1} \ldots c_{N_{3}}\right\rangle,
$$

which is the operator corresponding to the classical smeared variable $R_{f}=(1 / L) \int_{0}^{\infty} f R d r$, for suitable test functions $f$ - (the factor $L$ gives it dimension length). The conjugate momentum operator exists only in the exponentiated form in this quantization. Its action is

$$
\begin{aligned}
& e^{\left.i \widehat{\lambda_{j} P_{R}\left(x_{j}\right.}\right)}\left|a_{1} \ldots c_{N_{3}}\right\rangle \\
& =\left|a_{1} \ldots, a_{j}-\lambda_{j}, \ldots c_{N_{3}}\right\rangle
\end{aligned}
$$


where $a_{j}$ is 0 if the point $x_{j}$ is not part of the original basis state. In this case the action creates a new 'excitation' at the point $x_{j}$ with 'mode' $-\lambda_{j}$. These definitions give a faithful representation of the classical Poisson bracket of these variables. The Poisson bracket commutator correspondence fixes $L=\sqrt{2} l_{P}$, where $l_{P}$ is the Planck length. Similar definitions and the commutator result hold for the other phase space variables $\Lambda_{f}, \exp \left(i P_{\Lambda} / L\right)$ and $\phi_{f}, \exp \left(i L^{2} P_{\phi}\right)$, where the corresponding operators act on the basis state excitations $b_{1} \ldots b_{N_{2}}$ and $c_{1} \cdots c_{N_{3}}$ respectively. In the following we suppress the scalar field part of the basis states, since these are not needed for defining operator analogs of $\theta_{ \pm}$.

With the basic operators defined, we are in a position to address the question of the horizon operators. This requires defining operators for $P_{\Lambda}(r, t), R^{\prime}(r, t)$, and $\Lambda^{\prime}$ as a glance at Eqns. (10) shows. Since only translation operators (14) are available in our quantization, and these are not weakly continuous in the parameter translation parameter $\lambda$ [4], we define $P_{\Lambda}$ indirectly by

$$
\hat{P}_{\Lambda}^{\lambda}=\frac{l_{P}}{2 i \lambda}\left(\hat{U}_{\lambda}-\hat{U}_{\lambda}^{\dagger}\right)
$$

where $0<\lambda \ll 1$ is an arbitrary but fixed parameter, and $U_{\lambda}$ denotes $\exp \left(i \lambda P_{\Lambda} / L\right)$. This is motivated by the corresponding classical expression, where the limit $\lambda \rightarrow 0$ exists, and gives the classical function $P_{\Lambda} . \lambda$ is perhaps best understood as a ratio of two scales, $\lambda=l_{p} / l_{0}$, where $l_{0}$ is a system size. It is evident that momentum in this quantisation can be given approximate meaning only for $\lambda \ll 1$. $\lambda$ is also the minimum value by which an excitation can be changed.

Definitions for the operators corresponding to $R^{\prime}$ and $\Lambda^{\prime}$ are obtained by implementing the idea of finite differencing using the operator $\hat{R}_{f}$ (13). We use narrow Gaussians with variance proportional to the Planck scale, peaked at coordinate points $r_{k}+\epsilon l_{P}$, where $0<\epsilon \ll 1$ is a parameter designed to sample neighbouring points:

$$
f_{\epsilon}\left(r, r_{k}\right)=\frac{1}{\sqrt{2 \pi}} \exp \left[-\frac{\left(r-r_{k}-\epsilon l_{P}\right)^{2}}{2 l_{P}^{2}}\right]
$$

Denoting $R_{f_{\epsilon}}$ by $R_{\epsilon}$ for this class of test functions we define

$$
\hat{R}^{\prime}\left(r_{k}\right):=\frac{1}{l_{P} \epsilon}\left(\hat{R}_{\epsilon}-\hat{R}_{0}\right) .
$$

A further motivation of this form is that in the gauge $R=$ $r$ the corresponding classical expression gives unity in the limit $\epsilon \rightarrow 0$. This definition captures the simplest finite difference approximation to the derivative at the operator level. If a state has excitations separated by $\epsilon l_{P}$ then the classical intuition of the derivative is approximately implemented for that state. Such states may be viewed as potential "pre-semiclassical" states in the sense that they represent approximately differentiable field values, as opposed to "purely quantum" states which may be "nowhere differentiable" in the sense that they represent isolated excitations at large coordinate separations. The quantisation of $\Lambda^{\prime}$ is analogous to (17).

Apriori the parameters $\lambda$ and $\epsilon$ have no relation to each other since the former is an element of radial field excitation, and the latter is a coordinate separation. However, the classical falloff $R(r) \sim r$ for large $r$ suggests that it is plausible to set $\lambda=\epsilon$. Nevertheless we will keep them separate for the present discussion.

Lastly, we note that the classical marginal trapping conditions (4) are independent of the overall factor of $1 / \Lambda$ in (10), for $\Lambda>0$. A classically equivalent expression of these conditions is obtained by multiplying (10) by $1=\Lambda / \Lambda$, taking the numerator inside the bracket, and dropping the factor $1 / \Lambda^{2}$. This gives the classical form we utilise for the quantum theory.

Putting all these pieces together, we can construct the local operators

$$
\begin{aligned}
\hat{\theta}_{ \pm}\left(r_{k}\right)= & -\frac{2}{\epsilon l_{P}} \hat{R}_{0}^{2} \hat{\Lambda}_{0}^{2}\left(\hat{\Lambda}_{\epsilon}-\hat{\Lambda}_{0}\right) \mp \frac{l_{P}}{2 i \lambda}\left(\hat{U}_{\lambda}-\hat{U}_{\lambda}^{\dagger}\right) \hat{\Lambda}_{0} \\
& -\frac{4}{\epsilon l_{P}} \hat{\Lambda}_{0}^{3} \hat{R}_{0}\left(\hat{R}_{\epsilon}-\hat{R}_{0}\right),
\end{aligned}
$$

which have a well defined action on the basis states. We now use these operators to identify quantum black hole states.

A first observation is that our basis states are not eigenstates of $\hat{\theta}_{ \pm}$, since the $\hat{U}_{\lambda}$ are finite shift operators. One might be tempted to look for solutions of the condition $\hat{\theta}_{+}|\Psi\rangle=0$, which looks like a Dirac quantization condition. However, as in the latter case, the resulting solutions may not be normalizable states. For this reason we propose that a state $|\Psi\rangle$ represents a quantum black hole if

$$
\left\langle\Psi\left|\hat{\theta}_{+}\left(r_{k}\right)\right| \Psi\right\rangle=0, \quad\left\langle\Psi\left|\hat{\theta}_{-}\left(r_{k}\right)\right| \Psi\right\rangle<0 .
$$

for some $r_{k}$. The corresponding horizon size is given by

$$
R_{H}=\left\langle\Psi\left|\hat{R}_{0}\right| \Psi\right\rangle .
$$

This proposal gives a simple two step procedure for locating a quantum horizon: Given a state, one scans all its excitations using $\hat{\theta}_{+}\left(r_{k}\right)$ until a location is found where the conditions (19) hold. Once this is done, the physical horizon size is determined by computing the expectation value of $\hat{R}_{0}\left(r_{k}\right)$. This resembles the classical horizon location procedure.

It is apparent that the coordinate location of the quantum horizon $r_{k}$ is sharp in the sense that it is determined by the peak of a Gaussian. However, the horizon size is not sharp since the states satisfying the horizon conditions are not eigenstates of $\hat{R}_{f}$. As a consequence the horizon area is also not sharp. This has clear implications for the semiclassical entropy-area equation, which 
in light of this quantization will likely arise as an equality of expectation values.

Let us consider now the effect of the radial diffeomorphisms on the basis states. Denoting their action on coordinates by $\phi: r \longrightarrow \phi(r)$, the induced action on a basis element $\left|\mu_{1}\left(r_{1}\right) \cdots \mu_{N}\left(r_{N}\right)\right\rangle$ is

$$
\left.\hat{\phi}\left|\mu_{1}\left(r_{1}\right) \cdots \mu_{N}\left(r_{N}\right)\right\rangle\right)=\left|\mu_{1}\left(\phi\left(r_{1}\right)\right) \cdots \mu_{N}\left(\phi\left(r_{N}\right)\right)\right\rangle .
$$

From this it is clear that if a general state $|\Psi\rangle$ satisfies the horizon conditions with respect to $\hat{\theta}_{ \pm}(r)$ then $\hat{\phi}|\Psi\rangle$ will satisfy the condition for $\hat{\theta}_{ \pm}(\phi(r))$. It follows that the horizon coordinate location in the background manifold changes, while the horizon size remains the same. This mirrors exactly what happens at the classical level.

Explicit expressions for states satisfying the quantum black hole conditions are not hard to construct. A first example is the "vacuum" $|00 \cdots 00\rangle$ which has no excitations of any of the fields. It is easy to verify that for this state $\left\langle\hat{\theta}_{ \pm}\left(r_{k}\right)\right\rangle=0$ for all coordinates $r_{k}$, and that $\left\langle\hat{R}_{0}\left(r_{k}\right)\right\rangle=0$. The trapping evident in this state is trivial in the sense that the expectation values of the terms in $\hat{\theta}_{ \pm}$are each separately zero.

To construct a class of non-trivial black hole states, we note that $\hat{\theta}_{ \pm}$depends on the fixed parameters $\lambda, \epsilon$, and acts on field excitations at the coordinate values $r_{k}$ and $r_{k}+\epsilon l_{p}$. Specifically let

$$
\begin{aligned}
|\Psi\rangle= & \frac{1}{\sqrt{2}}\left(\left|\cdots a_{k}, a_{k+1}, \cdots ; \cdots, b_{k}, b_{k+1}, \cdots\right\rangle\right. \\
& \left.+i\left|\cdots a_{k}, a_{k+1} \cdots ; \cdots b_{k}-\lambda, b_{k+1}, \cdots\right\rangle\right)(21
\end{aligned}
$$

for excitations $a_{k}, a_{k+1}$ etc. at locations $r_{k}$ and $r_{k+1}$. For this state we have $\left\langle\hat{R}_{0}\left(r_{k}\right)\right\rangle=\sqrt{2} l_{P} a_{k}$, and

$$
\begin{aligned}
\frac{1}{l_{P}}\left\langle\hat{\theta}_{ \pm}\left(r_{k}\right)\right\rangle= & -\frac{8 a_{k}^{2}}{\epsilon}\left[b_{k}^{2} \delta b_{k}+\left(b_{k}-\lambda\right)^{2}\left(\delta b_{k}+\lambda\right)\right] \\
& -\frac{8 a_{k} \delta a_{k}}{\epsilon}\left[b_{k}^{3}+\left(b_{k}-\lambda\right)^{3}\right] \\
& \pm\left(\frac{b_{k}}{2 \lambda}-\frac{1}{4}\right)
\end{aligned}
$$

where $\delta a_{k}=a_{k+1}-a_{k}$, with the same for $b_{k}$. It is evident from this equation that states satisfying the quantum black hole conditions depend on the relative values of the excitations at neigbouring points. For illustration let us consider some specific cases. (i) $a_{k}=b_{k}=0$ : $\left\langle\theta_{+}\left(r_{k}\right)\right\rangle>0$ and $\left\langle\theta_{-}\left(r_{k}\right)\right\rangle<0$, so there is no horizon. (ii) $b_{k}=0$ : there is a value of $a_{k+1}\left(a_{k}, \lambda, \epsilon\right)$, given by a linear equation, for which $\left\langle\theta_{+}\left(r_{k}\right)\right\rangle=0$ and $\left\langle\theta_{-}\left(r_{k}\right)\right\rangle<0$. (iii) More generally, the quantum trapping condition $\left\langle\theta_{+}\left(r_{k}\right)\right\rangle=0$ may be viewed as a linear equation either for $a_{k+1}$, or for $b_{k+1}$, with all other corresponding values fixed. (iv) The generic state of this type represents normal untrapped space with no horizons because the third term in brackets in (22) is positive and large for $b_{k}$ of order unity since $\lambda \ll 1$. The $\hat{\theta}_{ \pm}$operators will have the same practical utility for semiclassical states, which arise as infinite linear combinations of the basis states, with a parameter specifying the classical mass.

Finally it is interesting to note that changing the state (21) by moving the $i$ factor from the second component to the first results in an overall sign change in the third term in (22), with no effect on the first two terms. This means that one can obtain white hole states satisfying $\left\langle\hat{\theta}_{+}\left(r_{k}\right)\right\rangle=0$ and $\left\langle\hat{\theta}_{-}\left(r_{k}\right)\right\rangle>0$. This shows the richness evident in the state space already at the two component level.

To summarise, let us recall the most prominent results of this work. We have constructed operator analogs of the classical null expansions, and used these to define the notion of a quantum black hole. The resulting quantum states are normalizable and thus bona fide elements of the Hilbert space. Our use of trapping conditions to define quantum black hole states corresponds to the classical dynamical trapping horizon introduced in [5], which applies to fully dynamical horizons. This is unlike the use of the isolated horizons [15] which are not applicable to dynamical situations. Furthermore, our quantization is in a setting that has been shown to be singularity free [4]. An important and physically appealing consequence of our results arises from the fact that the horizon operator does not commute with the area operator: the horizon size is subject to quantum fluctuations in the sense that it does not have sharply defined area eigenvalues.

Finally, it may prove valuable that our setup is close to the one used in numerical black hole calculations: the Hamiltonian evolution of a given initial state with no quantum horizon can be followed to horizon formation, and beyond. This analogy will facilitate numerical implementation of more involved calculations. The stage is thus set to study various dynamical processes of quantum black holes such as formation, mergers and evaporation. The first of these processes would be the quantum ana$\log$ of the classical black hole formation in this system studied in 14.

This work was supported in part by the Natural Science and Engineering Research Council of Canada. We thank Arundhati Dasgupta for comments on the manuscript.

* husain@math.unb.ca, oliver@math.unb.ca

[1] R. Narayan, astro-ph/0310692

[2] M. J. Rees, astro-ph/0401365

[3] For recent reviews see R. M. Wald, Living Rev. Rel. 4 (2001) 6 gr-qc/9912119; T. Padmanabhan, Phys. Rept. 406 (2005) 49-125 gr-qc/0311036.

[4] V. Husain, O. Winkler, gr-qc/0410125 Phys. Rev. D71, 104001 (2005) gr-qc/0503031. 
[5] Sean A. Hayward, Phys. Rev. D 49, 6467 (1994).

[6] B. K. Berger, D. M. Chitre, Y. Nutku, V. E. Moncrief, Phys. Rev. D5, 2467-2470(1971).

[7] S. W. Hawking, Commun. Math. Phys. 43 (1975) 199.

[8] W. Unruh, Phys. Rev. D14, 870 (1973).

[9] P. Hajicek, Phys. Rev. D30 1178 (1984).

[10] T. Thiemann and H. A. Kastrup, Nucl. Phys. B425, 665 (1994).
[11] K. Kuchar, Phys. Rev. D50, 3961 (1994).

[12] John. L. Friedman, Jorma Louko, Stephen N. WintersHilt, Phys. Rev. D56, 7674 (1997); gr-qc/9706051

[13] D. Grumiller, W. Kummer, D.V. Vassilevich, Phys. Rept. 369, 327 (2002); hep-th/0204253

[14] M. Choptuik, Phys. Rev. Lett. 70, 9 (1993).

[15] M. Bojowald and R. Swiderski, gr-qc/0410147 